\title{
EL MODELO TPACK EN LA FORMACIÓN INICIAL DE PROFESORES: MODELO UNIVERSIDAD DE PLAYA ANCHA(UPLA), CHILE.
}

\section{THE TPACK MODEL IN INITIAL TEACHER TRAINING: MODEL UNIVERSITY OF PLAYAANCHA (UPLA), CHILE}

\author{
Dr. Jaime Patricio Leiva Núñez ${ }^{1}$ \\ jleiva@upla.cl \\ Dra. Lastenia Ugalde Meza ${ }^{1}$ \\ lastenia.ugalde@upla.cl \\ Dra. Carmen Llorente-Cejudo ${ }^{2}$ \\ karen@us.es la Educación Chile.
Avenida Leopoldo Carvallo, 270. Valparaíso, Chile.
${ }^{2}$ Departamento de Didáctica y Organización Educativa, Facultad de Educación, Universidad de Sevilla
C/Pirotecnia s/n 41013, Sevilla. \\ ${ }^{1}$ Facultad de Ciencias Naturales y Exactas. Universidad de Playa Ancha de Ciencias de
}

Resumen: El nuevo modelo educativo de la Universidad de Playa Ancha, requiere incorporar sistemáticamente las TIC en el proceso de enseñanza, ya que considera prioritario el uso de nuevas metodologías que permitan al docente centrar su atención en la persona del estudiante y en sus aprendizajes, así como generar estudiantes que desarrollen competencias en el uso de TIC. Se genera un modelo para su incorporación en la formación inicial, basado en el modelo TPACK de Mishra (2006) y el concepto de entornos personales para el aprendizaje de Castañeda (2013). La puesta en práctica del modelo a partir del año 2015 revela resultados interesantes que se muestran en este trabajo.

Palabras clave: TPACK, tecnología educativa, competencias TIC, formación inicial, métodos de enseñanza.

Abstract: The new educational model of the University of Playa Ancha requires the systematic incorporation of ICT in the teaching process, since it considers it a priority to use new methodologies that allow the teacher to focus on the student's person and their learning, to generate autonomous students and Considers that these, should develop competences in the use of Tic. A model is generated to incorporate the Tic in the initial training based on the TPACK model Mishra (2006) and the concept of personal environments for the learning of Castañeda (2013). The implementation of the model from the year 2015 reveals interesting results that are shown in this work.

Keywords: TPACK, educational technology, ICT competences, initial training, teaching methods.

Píxel-Bit. Revista de Medios y Educación. No 53 Julio 2018. ISSN: 1133-8482. e-ISSN: 2171-7966. doi: http://dx.doi.org/10.12795/pixelbit.2018.i53.11 


\section{Introducción.}

Los objetivos del nuevo Modelo Educativo de la Universidad de Playa Ancha se orientan a formar profesionales comprometidos con la comunidad, con una sólida base disciplinar, con capacidad de análisis para enfrentar el desarrollo cultural y las transformaciones sociales propias de un mundo globalizado, y entre sus prioridades está la búsqueda de nuevas metodologías que permitan al docente centrar su atención en la persona del estudiante y en sus aprendizajes, por sobre los contenidos, poniendo en práctica estrategias flexibles y creativas, que potencien la resolución de problemas y el aprendizaje significativo. Así, la docencia se entiende como un proceso interactivo y no como la entrega unilateral de conocimientos específicos desvinculados de esta relación.

En esta búsqueda, la Universidad ha ido desarrollando diversos enfoques curriculares, transitando desde la modalidad centrada más en los contenidos y en métodos pedagógicos tradicionales, con sistemas de evaluación preferentemente sumativos, hacia un modelo que da paso a una nueva forma de concebir el proceso formativo, centrándose en la persona del estudiante y sus aprendizajes, demostrando competencias y resultados de aprendizaje con el uso de metodologías activas y participativas y con procesos evaluativos con criterios e indicadores coherentes con desempeños y aprendizajes esperados. (UPLA, Vicerrectoría Académica). Para poner en práctica el nuevo modelo educativo y de formación, se consideró partir en una primera etapa con todas las carreras de Pedagogía, y fue necesario llegar a un consenso sobre el perfil de egreso de los estudiantes de la UPLA, lo que se logró gracias al levantamiento de competencias sello, nucleares y disciplinares al interior de cada facultad, departamentos disciplinarios y carreras; proceso iniciado con el proyecto Mecesup UPA0802 (UPLA Vicerrectoría Académica) y la Dirección de Estudios y de Innovación Curricula. La formación del Sello institucional para el estudiante de la Universidad de Playa Ancha señala las siguientes competencias (UPLA, Vicerrectoría Académica):

-Compromiso ético: Elabora un marco de conducta ética que regula su actuación como estudiante y profesional en formación; Asume el compromiso ético de poner sus talentos y saberes al servicio de las personas con las que interactúa; demuestra capacidad para actuar en beneficio del bien común y lo hace de manera efectiva.

-Autovaloración: Logra la valoración de su ser y del otro como persona; promueve la inclusividad, el pluralismo y la tolerancia en la perspectiva de la observación y respeto a los derechos humanos; reconoce y explícita el derecho a la educación con igualdad de oportunidades para todos, siendo la diversidad un valor que enriquece y enaltece su tarea de formación profesional.

-Responsabilidad Social Universitaria: Asume responsablemente la ciudadanía y el compromiso social; proyecta su labor académica al medio socio-cultural, retroalimentando los procesos de formación académica en la Universidad.

-Analítico, crítico y proactivo: Demuestra su sentido analítico, crítico y proactivo; propone iniciativas que atienden necesidades del contexto profesional; innova en contextos profesionales complejos, dinámicos y diversos.

-Creativo: Busca e implementa soluciones nuevas a problemas derivados del contexto de su formación profesional; genera ideas 
innovadoras y asume los riesgos que comprende la implementación de éstas; demuestra originalidad, propone y conecta ideas, se organiza y se coordina para el trabajo individual $\mathrm{y} / \mathrm{o}$ en equipos.

-Liderazgo: Asume liderazgo en escenarios diversos con respuestas innovadoras y pertinentes, con el propósito de generar cambios en espacios diversos y mejorar los procesos de aprendizaje.

Las competencias instrumentales se refieren al desarrollo y dominio de habilidades asociadas a la condición de profesional en formación de nuestros estudiantes, y que se orientan a la vida universitaria y a facilitar el cumplimiento de tareas de auto-formación principalmente. La formación instrumental para el estudiante de la Universidad de Playa Ancha, señala competencias en las siguientes áreas:

-Comunicación: Utiliza la lengua materna en contextos académicos y profesionales; es capaz de comunicarse en un segundo idioma.

-Utilización de TIC: Usa TIC como recursos de trabajo; usa las TIC para trabajar en redes; usa las TIC para acceder a información.

En el contexto relacionado con las competencias instrumentales TIC, la universidad no dispone de ningún tipo de información que permita direccionar las intervenciones en esta área. Por esta razón, y para disponer de información precisa para planificar las actividades curriculares de las nuevas mallas, es que se ha desarrollado la presente investigación.

\subsection{Competencias TIC Docentes: algunas referencias.}

El problema principal en los sistemas educativos del siglo XXI es que la sociedad cambia rápida y continuamente, las tecnologías generan nuevas formas de aprender y los docentes se encuentran en una situación compleja ya que la estructura y gestión de las escuelas como las formas de enfrentar los procesos de aprendizaje no se han modificado mayormente. Los niños y jóvenes de este siglo necesitan otro tipo de formación. Los profesores se han formado y se están formando con una cultura y una visión del significado de su profesión que ya ha cambiado (Gros, 2005), tenemos entonces, que tomar conciencia como lo señala Prensky (2011) que construir aprendizaje en el siglo XXI usando las estructuras y restricciones del siglo XX es un propuesta absurda y temeraria que muchas veces fracasa. Por lo anterior, las instituciones de Educación Superior encargadas de la formación de profesores deben enfrentar el desafío de preparar docentes capaces de incorporar efectivamente el uso de los recursos tecnológicos en los procesos de aprendizaje. Para lograrlo se requiere adquirir nuevas destrezas y habilidades para el uso de las tecnologías y es fundamental para este proceso, desarrollar estándares que sirvan como guía para la implementación de las TIC en la formación docente. (UNESCO, División de Educación superior., 2004). Son varias las organizaciones que han definido estándares tic para profesores y también en algunos casos para estudiantes y directivos educacionales, entre ellas se pueden citar el ISTE (International Society Technology Education) de los estados Unidos, la QTS (Standard for the award of Qualified Teacher Status) del Reino Unido, la European Pedagogical ICT de la Comunidad Europea y otras organizaciones internacionales como la UNESCO. Los estándares TIC para docentes definidos por el ISTE se articulan 
alrededor de cinco ejes principales: (EDUTEKA, 2008):

Facilitan e inspiran el aprendizaje y la creatividad de los estudiantes.

Diseñan y desarrollan experiencias de aprendizaje y evaluaciones propias de la Era Digital.

Modelan el Trabajo y el Aprendizaje característicos de la Era Digital.

Promueven y Ejemplifican Ciudadanía Digital y Responsabilidad.

Se comprometen con el Crecimiento Profesional y con el Liderazgo.

En Chile, la primera definición de estándares TIC para la Formación Inicial Docente (FID), fueron fruto de un proyecto desarrollado por el Centro de Educación y Tecnología Enlaces del Ministerio de
Educación de Chile, con el apoyo de UNESCO. El proyecto nace del estudio realizado por profesionales del Centro Comenius de la Universidad de Santiago de Chile y de la Pontificia Universidad Católica de Valparaíso. Los Estándares se construyeron a partir de: a) una revisión bibliográfica de estándares TIC en la formación docente en el ámbito internacional considerando las experiencias Estadounidense, Europea, Latinoamericana y Australiana; b) la aproximación a «estándares» propiciada por la actividad de formación permanente de docentes impulsada en Chile por la Red Enlaces; c) el análisis de algunos programas de estudios de los itinerarios para formación de docentes de algunas Universidades del país y d) la

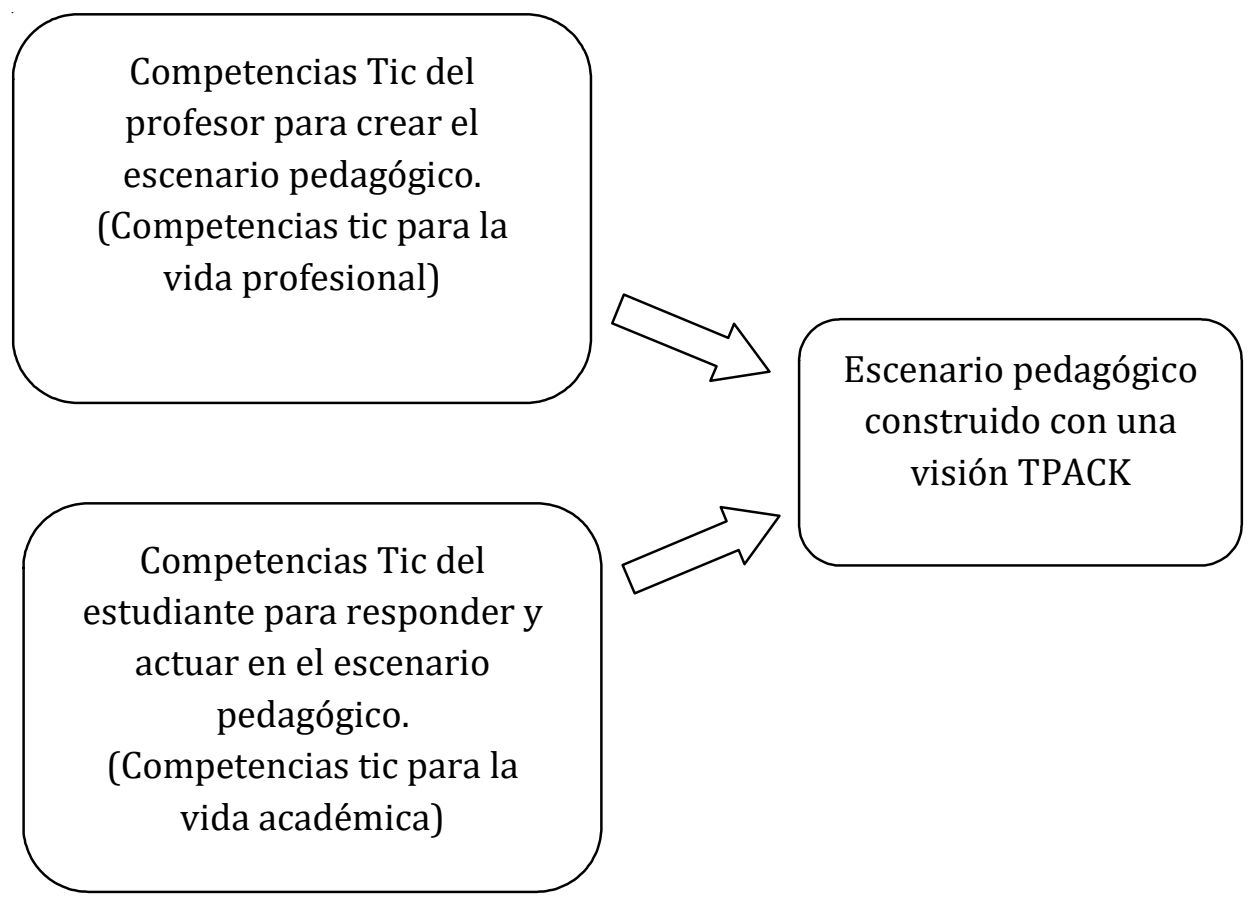

Figura 1. Módulos TIC para la formación de profesores UPLA. 
validación de los estándares por parte de una mesa de expertos.

\section{Desarrollo de la investigación.}

\subsection{Modelo TPACK/Modelo UPLA.}

En los últimos años han surgido diversidad de investigaciones relacionadas con el modelo TPACK de Mishra y Koehler (2006) orientadas a entender el rol de las tecnologías en el sistema educativo, las competencias docentes en TIC y la relación de las tecnologías con los otros dos componentes del modelo además de todos los constructos que emergen de él. El modelo TPACK (Technological Pedagogical Content Knowledge) de Mishra (2006) ha sido desarrollado a partir del modelo PCK (Pedagogical Content Knowledge) formulado por Shulman (1986). Este último modelo considera que el profesor se distingue de los especialistas del contenido porque en su práctica debe combinar el conocimiento de este contenido con los conocimientos pedagógicos. El modelo de Mishra (2006) agrega al modelo el conocimiento tecnológico que de alguna forma ha modificado la forma de hacer las cosas en la sociedad del conocimiento y por supuesto el proceso de enseñanza aprendizaje no ha sido la excepción.

El modelo TPACK de Mishra (2006) considera «Conocimiento Pedagógico» porque se refiere específicamente al trabajo del profesor, el «Conocimiento del Contenido» y el «Conocimiento Tecnológico». El modelo educativo por competencias de la UPLA (Universidad de Playa Ancha), considera igualmente estos tres componentes en las mallas curriculares de las carreras de pedagogía, sin embargo, no considera el término «conocimiento» sino que el término «competencia»: «Competencias Nucleares», «Competencias Disciplinares» $\mathrm{y}$ «Competencias Sello TIC».

En este sentido, Cabero, Marín y Castaño (X), ya hacía alusión al auge del citado modelo en esferas anglosajonas, asiáticas, australianas y europeasn (Albiom, JamiesonProctory Finger, 2010; Hsu, 2010; Jimoyiannis, 2010; Jang y Tsai, 2013; Kaya, Emre y Kaya, 2013; Liang, Chai,Ching, Koh, Jang y Tsa, 2013; Roig y Flores, 2014), hace que nos planteemos la necesidad de verificar si en el ámbito español y latinoamericano, tendría efectividad la aplicación del mismo. La importancia que en los últimos tiempos está adquiriendo este modelo para la formación y el perfeccionamiento del profesorado, nos ha llevado a traducir y fiabilizar un instrumento para el diagnóstico del modelo TPACK, seleccionando para ello el elaborado por Schmidt y otros (2009), entre otros motivos, además de por su tipo de construcción y facilidad de aplicación, porque es uno de las más utilizados para el diagnóstico del TPACK de un docente (Abbitt, 2011; Stewart, Antonenko, Shane, y Muravita, 2013; Roig y Flores, 2014; Mouza, Karchmer-Klein, Nandakumar, Ozden y Likun, 2014).

Desde esta visión del modelo TPACK, surgen los dos módulos o asignaturas que serán incorporadas en la malla curricular de las carreras pedagógicas de la UPLA. «Competencias tic la vida académica»y «Competencias tic para la vida profesional». En la figura 1, se muestra de manera esquemática la relación de los dos módulos propuestos en el modelo con el escenario pedagógico creado bajo la visión del modelo TPACK. 


\subsection{Módulo I «Competencias TIC para la} vida académica».

A través de este módulo, los estudiantes de pedagogía podrán adquirir o mejorar las competencias Tic necesarias para enfrentar como alumnos los desafíos de los nuevos escenarios de aprendizaje que deberán transitar en su proceso de formación. El análisis de las competencias TIC para ambos módulos puede abordarse e interpretarse desde varias perspectivas, sin embargo, en cualquier caso debemos partir señalando que debemos considerar por un lado un componente tecnológico y, por otro lado un componente informacional y/o comunicativo.

El componente de competencias Informacionales se ha definido en la comisión mixta (CRUE-TIC y REBIUM, 2009), como «el conjunto de conocimientos, habilidades, disposiciones y conductas que capacitan a los individuos para reconocer cuándo necesitan información, dónde localizarla, cómo evaluar su idoneidad y darle el uso adecuado de acuerdo con el problema que se les plantea».Por otra parte, en Information Literacy Competency Standards for Higher Education (American Library Association, 2000), se resalta el carácter general de estas competencia al considerar que son comunes en todos los ámbitos de estudio, en todos los entornos de aprendizaje y en todos los niveles educativos, además de lo anterior, estas competencias permiten dominar el contenido y ampliar las investigaciones, una mayor autonomía y la asunción de control del propio aprendizaje. Estas competencias involucran las siguientes habilidades:

-Búsqueda de la información necesaria.

-Análisis y selección de información de manera eficiente.
-Organización de la información adecuadamente.

- Utilización y comunicación de la información eficazmente de forma ética y legal, con el fin de construir conocimiento.

Desde esta perspectiva, si consideramos las competencias TIC informacionales, podemos encontrar una equivalencia entre ellas y los componentes definidos por Adell y Castañeda (Adell, 2010) para los Entornos Personales de Aprendizaje (PLE). Para ello, se estableció que el conjunto de herramientas que se incorporan en un PLE deben ser variadas, es por ello que se considera que un buen PLE debe contener (Castañeda, 2013):

-Herramientas, mecanismos y actividades para leer y acceder a la información

Herramientas: newsletters, blogs, canales video, lista de RSS, etc.

Mecanismos: búsqueda, curiosidad, iniciativa, etc.

Actividades: conferencias, lectura, revisión de titulares, visionado de audiovisuales.

-Herramientas, mecanismos y actividades para hacer/reflexionar haciendo:

Herramientas: blogs, cuaderno de notas, canal de video, sitio de publicación de presentaciones visuales, página web

Mecanismos: síntesis, reflexión, organización, estructuración, etc.

Actividades: creación de un diario de trabajo, hacer un mapa conceptual, publicar un vídeo propio, etc.

-Herramientas, mecanismos y actividades para compartir y reflexionar en comunidad. La PLN (Red Personal de Aprendizaje)

Herramientas: software social, seguimiento de la actividad en red, sitios de red social. 


\begin{tabular}{|c|c|}
\hline $\begin{array}{l}\text { Entornos Personales para el Aprendizaje } \\
\text { (Adell) }\end{array}$ & $\begin{array}{c}\text { Competencias Tic (Componente } \\
\text { Informacional) }\end{array}$ \\
\hline $\begin{array}{l}\text { - Herramientas, mecanismos } \\
\text { actividades para leer }\end{array}$ & - Búsqueda de la información necesaria \\
\hline $\begin{array}{l}\text { - Herramientas, mecanismos y } \\
\text { actividades para hacer/reflexionar } \\
\text { haciendo }\end{array}$ & $\begin{array}{l}\text { - Análisis y selección de información de } \\
\text { manera eficiente. } \\
\text { - Organización de la información } \\
\text { adecuadamente }\end{array}$ \\
\hline $\begin{array}{l}\text { Herramientas, mecanismos } \\
\text { actividades para compartir } \\
\text { reflexionar en comunidad }\end{array}$ & $\begin{array}{l}\text { - Utilización y comunicación de la } \\
\text { información eficazmente de forma } \\
\text { ética y legal, con el fin de construir } \\
\text { conocimiento }\end{array}$ \\
\hline
\end{tabular}

Tabla 1.Componentes de un PLE / Competencias Tic (componente informacional)

Mecanismos: asertividad, capacidad de consenso, diálogo, decisión, etc.

- Actividades: encuentros, reuniones, foros, discusiones, congresos, etc.

En la Tabla 1, se muestra un grado importante de correspondencia entre las habilidades descritas para el componente informacional de las competencias tic y el modelo de Adell y Castañeda para los entornos personales de aprendizaje.

\subsection{Módulo II «Competencias Tic para la vida profesional».}

Son varias las organizaciones que han definido las competencias TIC para profesores, $\mathrm{y}$ algunas de las que se mencionan son las siguientes (EDUTEKA, 2008):

Facilitan e inspiran el aprendizaje y la creatividad de los estudiantes.
Diseñan y desarrollan experiencias de aprendizaje y evaluaciones propias de la Era Digital.

- Modelan el Trabajo y el Aprendizaje característicos de la Era Digital.

- Promueven y Ejemplifican Ciudadanía Digital y Responsabilidad.

Se comprometen con el Crecimiento Profesional y con el Liderazgo.

\subsection{Modelo UPLA de competencias TIC en la formación de profesores.}

En la figura que se presenta (Figura 2) se establecen algunos elementos fundamentales a la hora de desarrollar y poner en práctica los módulos establecidos con anterioridad, por ejemplo que: Ambos módulos se trabajan en el segundo año del currículum de las carreras de pedagogía; El módulo 


\section{Modelo UPLA de competencias TIC en la formación inicial de los profesores}

\begin{tabular}{|c|c|c|c|}
\hline $\begin{array}{l}.0 \\
.0 \\
010 \\
0 \\
0 \\
0 \\
0 \\
0\end{array}$ & $\begin{array}{l}\text { Competencias } \\
\text { Tic del } \\
\text { profesor }\end{array}$ & $\begin{array}{l}\text { Módulo: } \\
\text { Competencias } \\
\text { Tic para la } \\
\text { vida } \\
\text { profesional }\end{array}$ & $\begin{array}{l}\text { PLE, búsqueda y procesamiento de } \\
\text { información digital, producir diferentes } \\
\text { formatos de documentos académicos, } \\
\text { comunicar utilizando medios digitales, } \\
\text { compartir y trabajar en forma } \\
\text { colaborativa }\end{array}$ \\
\hline F & $\begin{array}{l}\text { Competencias } \\
\text { Tic del } \\
\text { estudiante }\end{array}$ & $\begin{array}{l}\text { Módulo: } \\
\text { Competencias } \\
\text { Tic para la } \\
\text { vida } \\
\text { académica }\end{array}$ & $\begin{array}{l}\text { Generar material multimedia, trabajar } \\
\text { colaborativamente en ambientes } \\
\text { digitales, generar materiales } \\
\text { educativos interactivos desarrollo de } \\
\text { proyectos de innovación con Tic en su } \\
\text { ámbito profesional }\end{array}$ \\
\hline
\end{tabular}

Figura 2. Modelo UPLA: TIC en la formación inicial de profesores.

«Competencias Tic para la vida académica» se trabaja el primer semestre del segundo año; El módulo «Competencias Tic para la vida profesional se trabaja el segundo semestre del segundo año; Ambos módulos tienen una duración de un semestre académico (18 semanas); El trabajo directo semanal para ambos módulos es de dos sesiones; Ambos módulos son esencialmente prácticos y se trabajan en laboratorios de computación, considerando un estudiante por computador.

Toda la información de los módulos se puede obtener en www.cienciastic.cl/sello-tic

\section{Resultados}

Los dos módulos se realizaron por primera vez el año 2015 a la promoción de estudiantes que ingresaron a las carreras de pedagogía el 2014. Tal como se muestra en la Tabla 2, se pueden observar el total de carreras y el número de estudiantes de la primera promoción que desarrolla los módulos TIC.

Cabe señalar que se realizaron un total de 37 proyectos que involucraron un total de 144 estudiantes. La Facultad de Ciencias de la Educación lidera con un total de 14 proyectos y un total de 63 estudiantes involucrados. El contenido de estos proyectos se pueden revisar en sus respectivos blog en http://cienciastic.cl/ muestra2015/proyectos-2015/, donde se muestran ordenados por facultades. En general, podemos señalar que la mayoría de los proyectos tienen relación con colaborar directamente con las comunidades escolares en una diversidad de aspectos que van desde lo netamente académico hasta aspectos que tienen que ver con nutrición y ejercicio en escolares.

Durante el segundo semestre del 2016 generamos un convenio con Fundación Telefónica y se invitó a la primera generación 


\begin{tabular}{|l|c|}
\hline Carrera & Alumnos \\
\hline Ped. Castellano & 37 \\
\hline Ped.En Historia Y Geografía & 43 \\
\hline Ped. E. Física Damas & 32 \\
\hline Ped. E. Física Varones & 37 \\
\hline Ped. Filosofía & 12 \\
\hline Ed. Parvularia & 22 \\
\hline Ped. Ed. Musical & 47 \\
\hline Ped. Artes Plásticas & 26 \\
\hline Ped. Inglés & 37 \\
\hline Ped. Ed. Diferencial & 39 \\
\hline Ped. Ed. Básica & 39 \\
\hline Ped. Biología & 17 \\
\hline Ped. Química & 422 \\
\hline Ped. Física & \\
\hline Ped. Matematica & \\
\hline Total Alumnos & \\
\hline
\end{tabular}

Tabla 2, Carreras y números de alumnos 


\begin{tabular}{|l|l|}
\hline \multicolumn{2}{|l|}{ Curso Telefónica } \\
\hline Carrera & Alumnos \\
\hline Ed. Básica & 10 \\
\hline Biología & 6 \\
\hline Ed. & \\
Diferencial & 6 \\
\hline Matemática & 5 \\
\hline Química & 1 \\
& 28 \\
\cline { 2 - 2 } &
\end{tabular}

Tabla 3. Alumnos en curso on line.

de estudiantes que cursaron los módulos Tic durante el año 2015, a participar de una actividad de formación en modalidad BLearning, a través de la plataforma ScolarTIC. En esta actividad la propuesta fue realizar un módulo de «Programación Nivel Inicial» que incluyó, programación Scratch y App Inventor. En la Tabla 3, se muestran los datos de los estudiantes que se inscribieron en la actividad voluntaria, llegando a un total de 28 estudiantes.

\section{Conclusiones}

El modelo TPACK de Mishra resulta de gran utilidad cuando se requiere comprender el rol de las tecnologías en los sistemas educativos y demuestra tener una gran flexibilidad ya que permite desarrollar una gran variedad de consideraciones. En nuestro caso nos ha servido para sentar las bases para un modelo de incorporación de las tecnologías en la formación inicial docente de los futuros profesores. Los resultados obtenidos con la primera generación de estudiantes que desarrollaron los módulos Tic durante el año 2015, consideramos que ha sido exitosa, ya que los resultados demuestran que se ha generado un interés por usar las tecnologías y pensar en ellas como una herramienta importante para la vida profesional. Los 37 proyectos generados por los estudiantes, los seis proyectos que fueron presentados a congreso durante el año 2016, lo que significa que esos estudiantes han continuado trabajando en el tema por 
motivación propia y los 20 estudiantes que se inscriben voluntariamente en el perfeccionamiento con Fundación Telefónica, así lo demuestran. El trabajo realizado durante el 20015, ha permitido al grupo de profesores que trabajan en el proyecto afinar una serie aspectos sensibles y seguramente los resultados de los estudiantes del año 2016, mejorarán. Esto porque se nota un avance en la calidad y variedad de los trabajos. Se pueden obsrvar y comparar estos trabajos en las siguientes url: http://cienciastic.cl/ muestra2015/ y http://www.cienciastic.cl/ muestra2016.

\section{Referencias Bibliográficas}

Adell, S. (2010). Los entornos virtuales de aprendizaje (PLES): Una nueva manera de entender el aprendizaje. Recuperado de https:/digitum.um.es/jspui/bitstream/10201/ 17247/1/Adell\&Casta\%C3\%B1eda_2010.pdf (12-10-2011)

Abbitt, J. (2011). Measuring Technological Peda- gogical Content Knowledge in Preservice Teacher Education: A Review of Current Methods and Instruments, Journal of Research on Technology in Education, 43(4), pp. 281-300. Recuperado de http:// www.dlc-ubc.ca/word-press_dlc_mu/ educ500/files/2011/06/abbitt.pdf (12-10-2016)

Albiom, P., Jamieson-Proctor, R. \& Finger, G. (2010). Auditing the TPACK confidence of Australian preservice Teachers: The TPACK Confidence Survey (TCS). En C.D. Maddux, D., Ginson y B. Dodge (Eds.) Research highlights in technologu and teacher education 2010. Chesapeak: Society for Information Technology and Teacher Education (SITE), pp. 13-21.

American Library Association. (2000). Information Literacy Competency
Standards. Recuperado de https:// arizona.openrepository.com/arizona/ bitstream/10150/105645/1/standards.pdf (1003-2017)

Bauman, Z. (2007). Tiempos líquidos. Tusquets Editores.

Cabero, J. (2014). La formación del profesorado en TIC:Modelo TPACK. Sevilla: Secretariado de Recursos Audiovisuales y Nuevas Tecnologías de la Universidad de Sevilla.

Cabero, J., Marín, V. \& Castaño, C. (2015). Validación de la aplicación del modelo TPACK para la formación del profesorado en TIC. @tic. revista d'innovació educativa, 14. Recuperado de https://ojs.uv.es/index.php/ attic/article/view/4001/6235 (08-05-2017)

Cabero, J. (2001). Tecnología educativa. Diseño y utilización de medios en la enseñanza. Barcelona: Paidós.

Cabero, J., \& Barroso, J. (2015). Nuevos retos en tecnología educativa. Madrid: Editorial Síntesis.

Castañeda, L. (2013). Entornos personales de aprendizaje: Claves para el ecosistema educativo en red. Alcoy: Marfil, S.A.

Cejas, R. (2016). Las competencias del profesorado universitario desde el modelo TPACK (Conocimiento tecnológico y pedagógico del contenido). Pixel-bit Revista de Medios y Educación (49), 105-1019.

Cejas, R. (2016). Las competencias del profesorado universitario desde el modelo TPACK Conocimiento tecnológico y pedagógico del contenido, 49, 105-119.

Cox. S., G. (2009). Diagramming TPACK in practice: Using an elaborated model of the tpack framework to analyze and depict teacher knowledge. TechTrends , 53 (5), 60-69.

CRUE-TIC y REBIUM. (2009). Competencias informáticas 
informacionales en los estudios de grado. Recuperado de http://www.uv.es/websbd/ formacio/ci2.pdf(25-10-2016)

EDUTEKA. (2008). Estándares Nacionales (EEUU) de Tecnologías De Información Y Comunicación (Tic) Para Docentes. Recuperado de http://www.eduteka.org/ pdfdir/EstandaresNETSDocentes2008.pdf (14-5-2017)

Gros, B. (2005). La formación del profesorado como docente en los Espacios virtuales de aprendizaje. Recuperado de http://www.rieoei.org/deloslectores/ 959Gros.PDF (26-01-2016)

Hsu, S. (2010). Developing a scale for teacher integration of information and communication technology in grades 1-9. Journal of Computer Assisted Learn- ing, 26(3), pp.175-189. Recuperado de http:// onlinelibrary.wiley.com/doi/10.1111/j.13652729.2010.00348.x/pdf Doi: 10.1111/j.13652729.2010.00348.x

Jang, S \& Tsai, M. (2013). Exploring the TPACK of Taiwanese secondary school science teachers using a new contextualized TPACK model. Australasian Journal of Educational Technology, 29(4), pp. 566-580.

Jimoyiannis, A. (2010). Developing a Technological Pedagogical Content Knowledge Framework for Science Education: Implications of a Teacher Trainers' Preparation Program. Proceedings of Informing Science \& IT Education Conference (InSITE), pp. 597-607.

Kaya, Z., Emre, I. \& Kaya, O. (2013). Adaptation of Technological Pedagogical Content Knowledge Scale to Turkish. Theory \& Practice, volumen 13 (4), pp. 2367-2375.

Kozak, D., \& Lion, K. (2005). Redes y escuela: ¿Dentro o fuera? falsos dilemas sobre las TICS y su influencia en niños/as y jóvenes. Recuperado de http:// sedici.unlp.edu.ar/bitstream/handle/10915/ $\begin{array}{llllll}2 & 4 & 3 & 8 & 9\end{array}$ Documento_completo.pdf?sequence $=1$ (0302-2017)

Liang, J., Chai,Ching, S., Koh, J., Jang, C.\& Tsai, C. (2013). Surveying in service preschool teachers' technological pedagogical content knowledge. Australasian Journal of Educational Technology, 29(4), pp. 581-594.

Mishra, P. (2006). Technological pedagogical content knowledge: A framework for teacher knowledge. Teachers College Record, 108 (6), 1017-1054.

Mouza, C., Karchmer-Klein, R., Nandakumar, R., Ozden, O. \& Hu, L. (2014). Investigating the impact of an integrated approach to the development of preservice teachers' technological pedagogical content knowledge (TPACK). Computers \& Education, 71, pp. 206-221.

Prensky, M. (2011). Enseñar a nativos digitales. Madrid: SM.

OECD. (2008). New Millennium Learners: Initial findings on the effects of digital technologies on school-age learners. Recuperado de http://www.oecd.org/site/ educeri21st/40554230.pdf(24-06-2016)

Roig, R. \& Flores, C. (2014). Conocimiento tecnológico, pedagógico y disciplinario del profesorado: el caso de un centro educativo inteligente. EDUTEC, Revista Electrónica de Tecnología Educativa, 47. Recuperado de http://edutec.rediris.es/Revelec2/Revelec47/ n47_Roi g-Flores.html (13-10-2016)

Shulman, L. S. (1986). Those Who Understand: Knowledge Growth in Teaching. Educational Researcher, 15 (2), 4-14.

Stewart, J., Antonenko, P., Robinson, S., \& Muravita, M. (2013). Intrapersonal Factors Affecting Technological Pedagogical Content Knowledge of Agricultural Education Teachers. Journal of Agricultural 
Education, 54(3), pp. 157-170. Recuperado de http://www.jae-online.org/attachments/ article/1- 765/2012-0710\%20stewart1.pdf(0202-2016) Doi: 10.5032/jae.2013.03157

UNESCO, División de Educación superior. (2004). Las tecnologías de la Información y Comunicación en la formación Docente: Guía de Planificación. Recuperado de http:/ /unesdoc.unesco.org/images/0012/001295/ 129533s.pdf(12-12-2016)

UPLA Vicerrectoría Académica. (n.d.). Proyecto de Innovación Curricular Universidad de Playa Ancha. Recuperado de http://www.upla.cl/innovacioncurricular/ w p - c on t e n t / u p lo a d s / 2013 / 04 / PRESENTACI\%C3\%93N-PROYECTO-DEIC2013.-M.-L\%C3\%93GICO-11-junio.pdf (1403-2017)

UPLA, Vicerrectoría Académica. (n.d.). Modelo educativo Universidad de Playa Ancha. . Recuperado de http://www.upla.cl/ inicio/2012_0327_modelo_educativo.pdf(0701-2017)

Fecha de recepción: 27-10-2017

Fecha de evaluación: 18-12-2017

Fecha de aceptación: 18-12-2017 